\title{
Top squark with mass close to the top quark
}

\author{
Matthew R. Buckley, ${ }^{1}$ Tilman Plehn, ${ }^{2}$ and Michael J. Ramsey-Musolf ${ }^{3,4}$ \\ ${ }^{1}$ Department of Physics and Astronomy, Rutgers University, Piscataway, New Jersey 08854, USA \\ ${ }^{2}$ Institut für Theoretische Physik, Universität Heidelberg, Germany \\ ${ }^{3}$ Amherst Center for Fundamental Interactions, Department of Physics, University of Massachusetts, \\ Amherst, Massachusetts 01003, USA \\ ${ }^{4}$ Kellogg Radiation Laboratory, California Institute of Technology, Pasadena, California 91125, USA
}

(Received 20 April 2014; published 24 July 2014)

\begin{abstract}
The most natural supersymmetric solution to the hierarchy problem prefers the scalar top partner to be close in mass to the top quark. Experimental searches exclude top squarks across a wide range of masses, but a gap remains when the difference between the masses of the stop and the lightest supersymmetric particle is close to the top mass. We propose to search for stops in this regime by exploiting the azimuthal angular correlation of forward tagging jets in (s)top pair production. As shown in earlier work, this correlation is sensitive to the spin of the heavy states, allowing one to distinguish between top and stop pair production. Here, we demonstrate that this angular information can give a statistically significant stop pair production signal in the upcoming LHC run. While the appropriate simulation including parton showering and detector simulation requires some care, we find stable predictions for the angular correlation using multijet merging.
\end{abstract}

DOI: $10.1103 /$ PhysRevD.90.014046

PACS numbers: 14.65.Ha, 14.80.Ly

\section{INTRODUCTION}

The top partner holds a special place in many extensions of the standard model [1]. As the fermion with the largest coupling to the Higgs field, the top gives the largest quadratic correction to the Higgs mass term. To have a natural and untuned cancellation of this term, we would expect the supersymmetric top squark - the stop $(\tilde{t})$ - to be close in mass to the top itself. Additionally, in generic supersymmetric flavor models the large top Yukawa drives the mixing of left- and right-handed stops and pushes the lightest stop mass eigenstate to be the lightest squark. Experimentally, however, no evidence of a relatively light stop has been obtained in collider searches. A combination of ATLAS [2] and CMS [3] results at 7 and $8 \mathrm{TeV}$ excludes stop pair production decaying to final states containing an invisible, stable supersymmetric particle (e.g., the lightest neutralino, $\tilde{\chi}^{0}$ ) for stop masses in the range of $100-750 \mathrm{GeV}$, assuming a massless invisible decay product.

Nevertheless, in the two-dimensional plane of $\tilde{t}$ and $\tilde{\chi}^{0}$ masses, there remains a notable window in the experimental exclusion regions: neither experiment has ruled out the possibility that stop pair production events may be buried in top production when the mass difference $m_{\tilde{t}}-\left(m_{\tilde{\chi}}+m_{t}\right)$ becomes small. There is a simple explanation for this lack of sensitivity to stop production near the "degeneracy line": when the mass splitting is small, the invisible particles $\left(\tilde{\chi}^{0}\right)$ carry little momentum, so the final state from stop pair production closely mimics that of top pairs in the standard model. In principle, measurable differences in the missing transverse energy $\left(E_{T}\right)$ distributions would for fully hadronic top decays would appear if stop events are also present, a feature that might allow discovery or exclusion of degenerate stops [4]. In practice, however, such searches face challenging jet combinatorics and require precise understanding of the background $E_{T}$. In the dileptonic or semileptonic channels, kinematic variables built from the decay products of the top are nearly identical for $t \bar{t}$ and $\tilde{t}^{*}$ events, assuming the stop decays to either (a) an on-shell or off-shell top and an invisible $\tilde{\chi}^{0}$, (b) a bottom quark and a chargino, where the latter decays into a $\tilde{\chi}^{0}$ and a $W^{(*)}$ boson, or (c) the stop decays into a $\tilde{\chi}^{0}$ and a charm quark. This last search channel is of primary interest in the lowstop mass region, and is looked for in ATLAS and CMS using a charm-enhanced monojet-inspired search [5]. Analyzing differences in the top production angles or top decay products have been suggested [6] to search for stop pairs contaminating the top sample, but the possible improvement is small and can be washed out by necessary trigger and selection criteria.

In this study we explore an alternative approach for distinguishing top and stop pair production that avoids these difficulties. Specifically, we show how correlations between tagging jets can be used to search for stop pairs in the top pair sample at the LHC [7] independent of the stop decays. In particular, we consider the difference in the azimuthal angles $\Delta \phi$ of forward jets produced in association with the top or stop pair in vector boson fusion (VBF) events. ${ }^{1}$ These jets arise from initial state radiation. The information in their $\Delta \phi$ distribution can be used regardless of decay channels, as long as we can manage to extract a

\footnotetext{
${ }^{1}$ Here, the fusing vector bosons are primarily gluons, justifying the term "VBF."
} 
TABLE I. Cross sections for top and stop pair production at the $1.96 \mathrm{TeV}$ Tevatron and 7, 8, and $14 \mathrm{TeV}$ LHC. The theoretical predictions for the $t \bar{t}$ cross sections are calculated at NNLO + NNLL, for $m_{t}=173.3 \mathrm{GeV}$ [20]. Cross sections for stop pair production are calculated at NLO in PROSPINO2 [21] with a light $\tilde{t}_{1}$ and all other supersymmetric particles decoupled.

\begin{tabular}{|c|c|c|c|c|}
\hline$\sqrt{s}[\mathrm{TeV}]$ & $\sigma_{t \bar{t}}[\mathrm{pb}]$ Experiment & $\sigma_{t \bar{t}}[\mathrm{pb}]$ Theory & $\sigma_{\tilde{t} t^{*}}[\mathrm{pb}] m_{\tilde{t}}=175 \mathrm{GeV}$ & $\sigma_{\tilde{t} \tilde{t}^{*}}[\mathrm{pb}] m_{\tilde{t}}=200 \mathrm{GeV}$ \\
\hline 1.96 & $7.68 \pm 0.20_{\text {stat }} \pm 0.36_{\text {sys }}(\mathrm{CDF}+\mathrm{D} 0[19])$ & $7.164_{-0.200_{\text {scale }}^{+0.110}}^{+0.122_{\text {pdf }}}+0.169$ & 0.587 & 0.252 \\
\hline 7 & $\begin{array}{r}177 \pm 3_{\text {stat }} \pm_{7 \text { sys }}^{8} \pm 7_{\text {lumi }}(\text { ATLAS }) \\
\pm 3_{\text {stat }} \pm_{7}^{8} 7_{\text {sys }} \pm 7_{\text {lumi }}(\mathrm{CMS})\end{array}$ & $172.0_{-5.8_{\text {scale }}}^{+4.4}+4.8_{\text {pdf }}^{+4.7}$ & 24.0 & 11.9 \\
\hline 8 & $\begin{array}{c}238 \pm 2_{\text {stat }} \pm 7_{\text {sys }} \pm 7_{\text {lumi }} \pm 4_{\text {beam } E}(\text { ATLAS [17]) } \\
227 \pm 3_{\text {stat }} \pm 11_{\text {sys }} \pm 10_{\text {lumi }}(\operatorname{CMS~[18])}\end{array}$ & $245.8_{-8.4_{\text {scale }}^{+6.2}}^{+6.2}+6.4_{\text {pdf }}$ & 34.5 & 17.3 \\
\hline 14 & - & $953.6_{-33.9_{\text {scale }}^{+22.7}-17.8_{\text {pdf }}}^{+16.2}$ & 135 & 72.1 \\
\hline
\end{tabular}

signal-rich sample. As was originally demonstrated in the context of Higgs physics [8,9], the difference in azimuthal angle between the two forward jets $\Delta \phi$ from weak-bosonfusion events inherit information about the helicities of the weak bosons involved in the production. From the underlying argument it is obvious that this technique can be generalized to gluon fusion $[9,10]$. The helicities that can participate in a given process are set by the Lorentz structure of the production matrix element, and so for pair production the distribution of $\Delta \phi$ is sensitive to properties of pair-produced particles such as spin and $C P$ assignment.

For the pair production process of interest here, the resulting differential cross section has the form

$$
\frac{d \sigma}{d \Delta \phi}=A_{0}+A_{1} \cos \Delta \phi+A_{2} \cos (2 \Delta \phi)
$$

where the expansion coefficients $A_{k}$ encode the interplay of the underlying pair production amplitude and the helicity of the fusing gluons. As shown in our earlier work [11], the sign of $A_{2}$ is set by the spins of the produced particles: $A_{2}>0$ for scalars and $A_{2}<0$ for fermions. In general, this sensitivity could provide a powerful technique for diagnosing the spin of any new particles that may be discovered at the LHC [11]. This is also the case for top pair production close to threshold, while in the relativistic limit the sum of the two azimuthal angles is the more sensitive observable [7]. In the present context, we show how one may exploit the same effect to identify or exclude the presence of stop pairs in the region of parameter space near the degeneracy line. Moreover, we describe how the $\cos (2 \Delta \phi)$ correlation between initial state radiation jets can be reliably described in event simulations that take into account parton showering and realistic detector jet identification and show that the correlation is not washed out through azimuthal decorrelation $[12,13]$ To our knowledge, this study represents the first such demonstration, indicating that study of azimuthal tagging jet correlations may be a realistic tool in other contexts as well $[7,11]$.

Before determining if the degenerate stop production could be hiding in top pair production at the LHC, one should ask whether the measured cross section for top pair production allows for such a scenario, as in some of the earliest searches for stops at the Tevatron [14]. This rate has been measured numerous times [15-19] and agrees with theoretical predictions [20] within uncertainties. In Table I, we show the measured top pair cross sections at the Tevatron and the LHC, along with the theoretical predictions and the supersymmetric stop pair production cross sections for light stop masses of 175 and $200 \mathrm{GeV}$. At first glance, the measured cross section would appear to rule out the addition of a stop with mass near that of the top. However, it is unclear how the top cross section measurements would respond to an admixture of stop events, and there may be a degeneracy between the cross section and top mass measurements. Short of a detailed analysis of this question that goes beyond the scope of the present study, we cannot rule out the possibility-however unlikely-that a $175 \mathrm{GeV}$ stop could be hiding inside the top sample. Moreover, a stop with mass around $200 \mathrm{GeV}$, still within the degeneracy window, is not in significant tension with the experimental results, given the uncertainties. Consequently, we will consider two benchmark cases, corresponding to $\left(m_{\tilde{t}}, m_{\tilde{\chi}}\right)=$ $(175,1) \mathrm{GeV}$ and $(200,25) \mathrm{GeV}$, respectively.

Our discussion is organized as follows. In Sec. II we explain the physics behind the $\Delta \phi$ correlations of VBF tagging jets in the specific cases of top and stop pair production. In Sec. III we then discuss the simulation of these events including multijet merging in MADGRAPH5. While in the default setup the correlations between the tagging jets are not guaranteed to be included we show how they can be accounted for. In the same section we study the tagging jet correlations at parton level and show how a dedicated analysis can separate top and stop contributions to a mixed event sample. In Sec. IV we confirm that using realistic cuts and a fast detector simulation these results can be reproduced.

\section{TAGGING JET CORRELATIONS}

We are interested in top and stop pairs with two associated tagging jets, produced primarily via initial state radiation, or equivalently, through VBF diagrams [22]. 


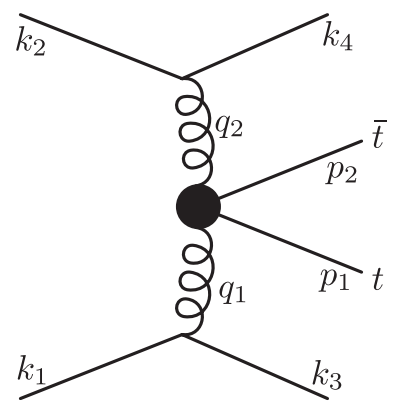

FIG. 1. A representative Feynman diagram for the VBF process $p p \rightarrow t \bar{t}+j j$ with two tagging jets. Similar diagrams exist for stop pair production. The initial and final state partons can be quarks, antiquarks, or gluons. The different channels contributing to the hard $g g \rightarrow t \bar{t}$ scattering are denoted by a solid dot.

Eventually, to separate VBF production from all other sources of jets we will employ strict selection cuts, primarily requiring the jets to be forward. A representative Feynman diagram is shown in Fig. 1, defining our notation for the different momenta. The full gauge-invariant matrix element will be the sum of many diagrams, but the cuts will emphasize this topology's contribution to the amplitude. In our simulations, we will include all initial parton states, though in practice gluons dominate for the parameter range of interest. It is most convenient to write the relevant kinematics in the three frames shown in Fig. 2 [8]. The emission of the fusing vector bosons (gluons in our case) from the incoming partons are described in the Breit frames (frames I and II), defined by the gluon momenta being purely spacelike and in the $z$ direction:

$$
\begin{aligned}
& q_{1}^{\mu}=k_{1}^{\mu}-k_{3}^{\mu}=\left(0,0,0, Q_{1}\right), \\
& q_{2}^{\mu}=k_{2}^{\mu}-k_{4}^{\mu}=\left(0,0,0,-Q_{2}\right) .
\end{aligned}
$$

The top/stop pair production frame shown as frame $\mathrm{X}$ in Fig. 2 is defined as the frame in which $q_{1}^{\mu}+q_{2}^{\mu}=(\sqrt{\hat{s}}, \overrightarrow{0})$, where $\hat{s} \equiv\left(p_{1}+p_{2}\right)^{2}$ is the invariant mass of the top or stop pair.

We now focus on the dependence of the differential cross section on the azimuthal angles $\phi_{1}$ and $\phi_{2}$. As long as the tagging jets with the momenta $k_{3}$ and $k_{4}$ are forward, the $z$ axis shared by frames I, II, and X is nearly collinear with

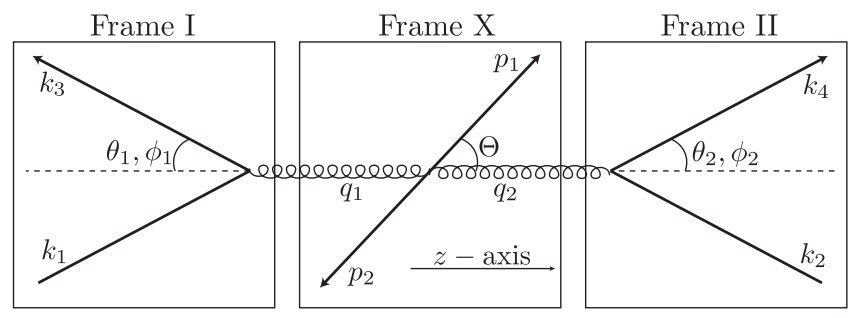

FIG. 2. Kinematics for VBF events, showing the two Breit frames I and II and the production frame X [8]. the experimental beam axis. In this approximation, an azimuthal rotation around the gluon momenta corresponds to a physical azimuthal rotation around the beam axis in the lab frame. Therefore, as a first step we can approximate the observed azimuthal angles in the laboratory frame by the angles in the plane orthogonal to the top or stop momenta [9], which identifies the angles $\phi_{1}$ and $\phi_{2}$ in the Breit frames with physical angles as measured by the detector. The validity of this approximation will be borne out in the results of the simulations, which do not make any such approximations. The matrix element for the full VBF event takes the form

$$
\mathcal{M}=\sum_{h_{1}, h_{2}} \mathcal{M}_{\mathrm{I}}^{\mu}\left(h_{1}, \phi_{1}, \theta_{1}\right) \mathcal{M}_{\mathrm{II}}^{\nu}\left(h_{2}, \phi_{2}, \theta_{2}\right) \mathcal{M}_{\mathrm{X}}^{\mu \nu}\left(h_{1}, h_{2}, \Theta\right),
$$

where $h_{1}, h_{2}=-1,0,+1$ are the helicities of the gluons $q_{1}$ and $q_{2}$, measured relative to the $z$ axis, so $h_{1}=+1$ is positive angular momentum for $q_{1}$, but $h_{2}=-1$ is positive angular momentum for $q_{2}$. We suppress the dependence on the color factors. A boost is required to take each matrix element from its individual frame to a common center-ofmass frame. All these boosts will be in $z$ direction and will not induce additional dependence on the azimuthal angles $\phi_{i}$. Therefore, $\phi_{1}$ and $\phi_{2}$ enter only as phases of the Breit matrix elements,

$$
\begin{aligned}
& \mathcal{M}_{\mathrm{I}}\left(h_{1}, \phi_{1}, \theta_{1}\right)=\mathcal{M}_{\mathrm{I}}\left(h_{1}, 0, \theta_{1}\right) e^{+i h_{1} \phi_{1}}, \\
& \mathcal{M}_{\mathrm{II}}\left(h_{2}, \phi_{2}, \theta_{2}\right)=\mathcal{M}_{\mathrm{II}}\left(h_{2}, 0, \theta_{2}\right) e^{-i h_{2} \phi_{2}} .
\end{aligned}
$$

We can rewrite $\phi_{1}$ and $\phi_{2}$ in terms of their difference $\Delta \phi \equiv$ $\phi_{1}-\phi_{2}$ and their sum $\phi_{+} \equiv \phi_{1}+\phi_{2}[8,9]$. The angle $\phi_{+}$is physically unobservable without reference to the top or stop production plane, which we will not attempt to reconstruct, and so it can be integrated over. Abbreviating the six-body phase space factors as $(\mathcal{P} S)$ and the integration over all other angles as $d \Omega$, the differential cross section with respect to $\Delta \phi$ can be written as

$$
\begin{aligned}
\frac{d \sigma}{d \Delta \phi}= & (\mathcal{P} S) \int d \Omega \sum_{h_{1}^{(\prime)}, h_{2}^{\left(\prime^{\prime}\right)}} e^{i \Delta h \Delta \phi / 2}\left[\mathcal{M}_{\mathrm{I}}^{\mu}\left(h_{1}\right) \mathcal{M}_{\mathrm{I}}^{\mu^{\prime} *}\left(h_{1}^{\prime}\right)\right] \\
& \times\left[\mathcal{M}_{\mathrm{II}}^{\nu}\left(h_{2}\right) \mathcal{M}_{\mathrm{II}}^{\nu^{\prime} *}\left(h_{2}^{\prime}\right)\right]\left[\mathcal{M}_{\mathrm{X}}^{\mu \nu}\left(h_{1}, h_{2}\right) \mathcal{M}_{\mathrm{X}}^{\mu^{\prime} \nu^{\prime} *}\left(h_{1}^{\prime}, h_{2}^{\prime}\right)\right],
\end{aligned}
$$

with $\Delta h=h_{1}-h_{1}^{\prime}+h_{2}-h_{2}^{\prime}$. This distribution has to be invariant under the shift $\Delta \phi \rightarrow \Delta \phi+2 \pi$, which translates into the condition $\Delta h=0, \pm 2, \pm 4$. Terms with odd $\Delta h$ must vanish, and larger values of $\Delta h$ cannot be generated for $\left|h_{j}\right| \leq 1$ (allowing for off-shell gluons). We then expand the exponential with the helicities in sines and cosines and, assuming $C P$ conservation, ignore the complex sine contributions. The three allowed helicity changes $\Delta h$ give rise to the three coefficients of Eq. (1), 


$$
\begin{aligned}
A_{n}= & (\mathcal{P} S) \int d \Omega \sum_{\Delta h= \pm n}\left[\mathcal{M}_{\mathrm{I}}^{\mu}\left(h_{1}\right) \mathcal{M}_{\mathrm{I}}^{\mu^{\prime} *}\left(h_{1}^{\prime}\right)\right] \\
& \times\left[\mathcal{M}_{\mathrm{II}}^{\nu}\left(h_{2}\right) \mathcal{M}_{\mathrm{II}}^{\nu^{\prime} *}\left(h_{2}^{\prime}\right)\right]\left[\mathcal{M}_{\mathrm{X}}^{\mu \nu}\left(h_{1}, h_{2}\right) \mathcal{M}_{\mathrm{X}}^{\mu^{\prime} \nu^{\prime} *}\left(h_{1}^{\prime}, h_{2}^{\prime}\right)\right] .
\end{aligned}
$$

We will be most interested in $A_{2}$, where $\Delta h= \pm 4$. This can only be satisfied by the unique configuration $h_{1}=h_{2}=$ \pm 1 and $h_{i}^{\prime}=-h_{i}$.

From explicit calculation, the contribution from the matrix elements for gluon emission, i.e. $\mathcal{M}_{\mathrm{I}}\left(h_{1}\right)^{\mu} \times$ $\mathcal{M}_{\mathrm{I}}\left(-h_{1}\right)^{\mu^{\prime} *}$ and $\mathcal{M}_{\mathrm{II}}\left(h_{2}\right)^{\nu} \mathcal{M}_{\mathrm{II}}\left(-h_{2}\right)^{\nu^{\prime} *}$ for $h_{i}= \pm 1$, are all positive [8]. As a result, the sign of $A_{2}$ depends only on the sign of the pair production interference terms $\mathcal{M}_{\mathrm{X}}^{\mu \nu}(h, h) \mathcal{M}_{\mathrm{X}}^{\mu^{\prime} \nu^{\prime} *}(-h,-h)$, with $h= \pm 1$. That is, the sign of $A_{2}$ depends on the relative sign between the matrix element for pair production where the total incoming $z$ component of angular momentum is +2 , and the matrix element where the incoming $J_{z}=-2$.

An explicit calculation of these interference terms in the case of the fusion of Abelian gauge bosons shows that, for the production of scalars, these interference terms are overall positive, while for fermion production, the terms are overall negative [11]. We can now repeat this calculation in the case of QCD-coupled heavy quarks [7] or squarks. The results are made more clear by multiplying the matrix elements in frame $\mathrm{X}$ by polarization vectors for the virtual gluons $q_{1}$ and $q_{2}$, treating them as approximately on shell. Recalling that positive helicity for both gluons is defined relative to the $z$ axis, rather than relative to the gluon momentum, both sets of polarization vectors can be written as $\epsilon_{1 / 2}^{ \pm}=(0,1, \pm i, 0) / \sqrt{2}$.

We begin with the fermionic case. For top pairs, the relevant production matrix elements times polarization vectors in frame $\mathrm{X}$ are

$$
\begin{gathered}
{\left[\mathcal{M}_{\mathrm{X}}^{\mu \nu}(h, h)\right]^{s, s} \epsilon_{\mu}(h) \epsilon_{\nu}(h)=-i g_{s}^{2} 2 s\left(\left\{T^{a}, T^{b}\right\}+\beta \cos \Theta\left[T^{a}, T^{b}\right]\right) \beta \sqrt{1-\beta^{2}} \frac{\sin ^{2} \Theta}{1-\beta^{2} \cos ^{2} \Theta},} \\
{\left[\mathcal{M}_{\mathrm{X}}^{\mu \nu}(h, h)\right]^{s,-s} \epsilon_{\mu}(h) \epsilon_{\nu}(h)=-h i g_{s}^{2} 2 s\left(\left\{T^{a}, T^{b}\right\}+\beta \cos \Theta\left[T^{a}, T^{b}\right]\right) \beta \sin \Theta \frac{1-2 \operatorname{sh} \cos \Theta}{1-\beta^{2} \cos ^{2} \Theta} .}
\end{gathered}
$$

The angle $\Theta$ is defined in Fig. 2. The superscripts $s, s$ or $s,-s$ for $s= \pm 1 / 2$ denote the helicities of the top and antitop, measured relative to each of their momenta. In terms of the total production energy $\hat{s}$ the velocity of the top and antitop $\beta$ is $\beta=\sqrt{1-4 m^{2} / \hat{s}}$.

Notably, the matrix elements for production of a $t \bar{t}$ pair with the same helicity assignments [Eq. (7)] do not have the property that $\mathcal{M}_{X}(+1,+1) \times \mathcal{M}_{\mathrm{X}}(-1,-1)^{*}<0$, contrary to our expectations. However, the signs of the $s,-s$ matrix elements with opposite helicity are manifestly asymmetric, as $\mathcal{M}_{\mathrm{X}}^{s,-s}(h, h) \propto h$, so this product is indeed negative. The fact that one term is not clearly negative could be concerning for our argument, but by inspection it is clear that the negative terms are strictly larger in magnitude than the positive contributions. It is possible that the $\beta$ dependence of the $A_{2}$ term could be useful in an experimental analysis. Cuts placed on the top decay products could be used to enhance particular ranges of $\beta$ [7], enhancing or suppressing the interference effect and providing useful sidebands. We will not further investigate this possibility in this paper.

Turning to the stop pair production, the relevant matrix elements are

$$
\begin{aligned}
\mathcal{M}_{\mathrm{X}}^{\mu \nu}(h, h) \epsilon_{\mu}(h) \epsilon_{\nu}(h)= & i g_{s}^{2}\left(\left\{T^{a}, T^{b}\right\}+\beta \cos \Theta\left[T^{a}, T^{b}\right]\right) \\
& \times \frac{\beta^{2} \sin ^{2} \Theta}{1-\beta^{2} \cos ^{2} \Theta} .
\end{aligned}
$$

Clearly this does not depend on the gluon helicities $h$, and so the interference terms are positive. This results in a positive $A_{2}$ term for stop pair production, and thus, the sign of $A_{2}$ can be used to distinguish the production of scalar stops and fermionic tops. Note that these two calculations only demonstrate that the top and stop distributions will have opposite signs of their $A_{2}$ components, without addressing the relative magnitudes. To answer that question, we must turn to Monte Carlo simulation.

\section{SIMULATING VBF (S)TOPS}

In order to extract information on the spin of the heavy top or stop particles from tagging jets we need to ensure that our simulation keeps all relevant spin correlations. Naively, this can be guaranteed by generating events for the hard processes $\tilde{t}^{*} j j$ and $t \bar{t} j j[7,11,23]$. However, the transverse momentum of the tagging jets will often be significantly below the energy scale of this hard process. In that region of phase space, for example the transverse momentum spectrum of jet radiation is only properly described once we include the parton shower or other implementation of Sudakov factors. In standard showering algorithms the probabilistic parton shower is (usually) averaged over the helicities of the participating partons. In such simulations, any apparent spin correlation between the hard process and the tagging jets-or between the tagging jets themselves - comes only from kinematic constraints [23], rather than from a combination of kinematics and underlying interference effects. What we need is a merged description of the parton shower and the hard 

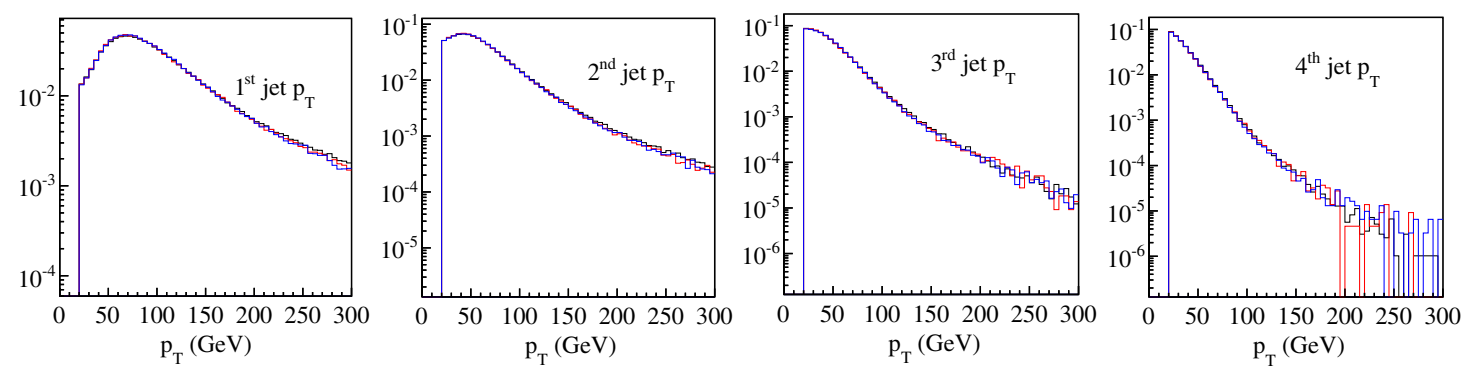

FIG. 3 (color online). Normalized $p_{T}$ distributions of the four leading jets in the merged $t \bar{t}$ samples, with XQCUT $=20$ (black), 40 (red), and $60 \mathrm{GeV}$ (blue). We use anti- $k_{T}$ jets with $\Delta R=0.5$, and require $p_{T}>20 \mathrm{GeV}$ and $\left|\eta_{j}\right|<5$.

matrix element, where the tagging jets are generated through the matrix element.

To that end, we consider two benchmark parameter points for stop signals for stop pair production followed by a decay into a top and a missing energy particle,

$$
p p \rightarrow \tilde{t} \tilde{t}^{*} \rightarrow\left(t \tilde{\chi}^{0}\right)\left(\tilde{t} \tilde{\chi}^{0}\right) \quad\left(m_{\tilde{t}}, m_{\tilde{\chi}}\right)=\left\{\begin{array}{l}
(175,1) \mathrm{GeV} \\
(200,25) \mathrm{GeV} .
\end{array}\right.
$$

The invisible particles coming from a prompt decay can be a neutralino or a gravitino. As we are not closely investigating the stop and top decay patterns we will refer to the generic missing energy particle as $\tilde{\chi}^{0}$.

For the background and each signal benchmark we generate events for the pair production of stops and tops at the $14 \mathrm{TeV}$ LHC with up to three extra jets in MAdGRAPH5 [24,25], matching the jets to PYTHIA6 [26] and using anti- $k_{T}$ jets with $R=0.5$ [27] down to a matching scale XQCUT $=20 \mathrm{GeV}$. This choice (endorsed by the MADGRAPH authors [28], and demonstrated in Refs. [29] to accurately model NLO effects) ensures that the spin correlations in the tagging jets are kept, provided the two tagging jets are chosen from the three leading jets that do not originate from top decay.

We will compare these results to unmatched hard $t \bar{t} j j$ and $\tilde{t t^{*}} j j$ events [7]. In this section we do not keep track of the top and stop decays. The two tagging jets are the two hardest jets which fulfill all $p_{T}$ and $\Delta \eta$ requirements.

In order to ensure that all final state jets in MADGRAPH5 are generated by the matrix element and hence include all spin and angular correlations, we can move the matching scale to values below the transverse momenta for all potential tagging jets, XQCUT $<p_{T, j}{ }^{2}$ While this choice will hugely decrease the efficiency of the event generation, because a very large fraction of events will be vetoed to generate the Sudakov suppression, it will ensure that our events include all the necessary information. Because the matching scale is not a physical parameter, it can be varied

\footnotetext{
${ }^{2}$ We have confirmed that for events with XQCUT $>p_{T, j}$ the correlations between the tagging jets in MADGRAPH are indeed lost.
}

within a reasonable range, where we will see that the definition of "reasonable" is different for kinematic distributions and the total rate.

Before we study the spin correlation between the tagging jets we test if our choice of the matching scale, $\mathrm{XQCUT}=20 \mathrm{GeV}$, leads to stable and consistent results. To this end we show the $p_{T}$ distributions for the first four jets for top pair production in Fig. 3. This distribution directly probes the Sudakov suppression and should therefore be most sensitive to artifacts from the choice of the matching scale. We vary the matching scale from 20 to $40 \mathrm{GeV}$ and the default value of $60 \mathrm{GeV}$. We see that the distributions are essentially indistinguishable between the three samples over the entire range of $p_{T}$, so our choice of scales does not present any problems for the tagging jet distributions.

On the other hand, the combined cross sections from MADGRAPH show a wider variation, with $\sigma_{t \bar{t}}=2.9,1.3$, 0.94 , and $0.71 \mathrm{nb}$ for XQCUT $=20,40,60$, and $100 \mathrm{GeV}$. Given that multijet merging is based on a combination of leading order matrix elements and a leading logarithmic parton shower, this variation reflects the uncertainty of a leading order cross section with four powers of $\alpha_{s}$. For smaller values of XQCUT we include more and more real emission as described by the full matrix element, but only compensated for by approximate virtual corrections in the Sudakov factor. If we apply an external normalization of the total production rate, for example to the precision predictions shown in Table I, we can use a MADGRAPH event samples with the matching scale of $20 \mathrm{GeV}$ to accurately simulate the production of top or stop pairs plus jets.

We can now consider the distribution of forward jets in top or stop events. In this section, we will focus on confirming the existence and the sign of the $A_{2}$ terms, as derived from the interference pattern described in Sec. II. Moreover, we need to test if our event generation indeed captures all relevant physics. To be independent of the details of the top decay, we use Monte Carlo truth to distinguish between associated jets and those from top decay. For specific top decays it should be straightforward to distinguish between ISR jets and decay jets, as has been shown for direct production of supersymmetric particles [30], for weak-boson-fusion pair production of supersymmetric particles [31], and for sgluon pair production [32], as 

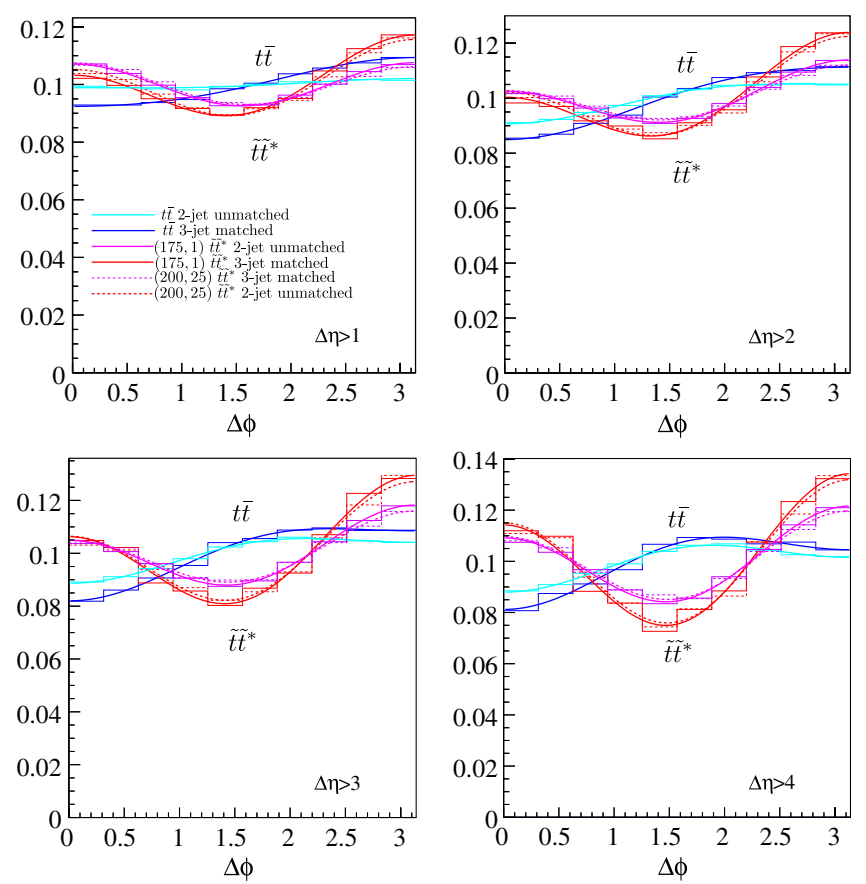

FIG. 4 (color online). Normalized $\Delta \phi$ distributions for the two highest- $p_{T}$ forward jets at parton level, requiring $\Delta \eta_{j j}>1,2,3,4$. We show top pairs (blue) and stop pairs (red) matched to three jets, as well as the unmatched two-jet samples for tops (cyan) and stops (purple). We also show the best fits to the functional form $A_{0}+A_{1} \cos \Delta \phi+A_{2} \cos (2 \Delta \phi)$. For the stop samples, the $\left(m_{\tilde{t}}, m_{\tilde{\gamma}}\right)=(175,1) \mathrm{GeV}$ scenario is shown with a solid line, while $\left(m_{\tilde{t}}, m_{\tilde{\chi}}\right)=(200,25) \mathrm{GeV}$ is shown with a dotted line.

we will demonstrate shortly. We then place selection criteria on our 3-jet matched or 2-jet unmatched samples in order to isolate VBF-type production from all other diagrams that generate two or more jets in association with stops or tops. Adapting the criteria used for $W$-boson fusion Higgs selection [8,31], we begin by requiring at least two parton-level jets in the merged sample with

$p_{T, j}>20 \mathrm{GeV}, \quad\left|\eta_{j}\right|<5, \quad \Delta \eta_{j j}>1,2,3,4$.

The increasing rapidity separation should emphasize the VBF-induced angular correlations between the tagging jets [9]. More realistic selection criteria will be put in place once we include a fast detector simulation in Sec. IV.

In Fig. 4 we plot the normalized $\Delta \phi$ distributions between the two highest- $p_{T}$ parton-level tagging jets defined in the laboratory frame, requiring $\Delta \eta_{j j}>1,2,3$, and 4 in the successive panels. As can be seen, there is a clear difference between the tagging jet correlations from stop and top events, corresponding to the sign of the $\cos (2 \Delta \phi)$ term. It induces a clearly visible minimum in the stop sample around $\Delta \phi=\pi / 2$, especially noticeable when compared to the slight excess here in the top sample. Top pairs are dominated by a slight preference for back-to-back tagging jets.

Without a $\Delta \eta_{j j}$ cut, the nontrivial azimuthal dependence would be highly suppressed. This is expected, since central jets do not predominantly come from the initial state radiation (ISR) diagrams and do not reflect information about the helicity of fusing gluons through interference patterns in our reference frame. As we enforce increasingly large $\Delta \eta_{j j}$ cuts we see a finite $\cos (2 \Delta \phi)$ component develop in both the top and stop samples; with the appropriate signs for fermionic and scalar pairs.

In Table II, we show the relative size of the $\cos \Delta \phi\left(A_{1}\right)$ and $\cos (2 \Delta \phi)\left(A_{2}\right)$ modes for the top background and stop benchmark points, normalized to the constant term $A_{0}$. The coefficients are obtained from the normalized ten-bin histograms at parton level, using the standard ROOT fitting algorithm. It is apparent that the nontrivial $A_{2}$ term is present in the unmatched two-jet sample, and survives after the addition of a third jet in the matching scheme. The magnitude of the $A_{1}$ term significantly increases for the matched samples.

Comparing the events with three merged jets and the events with only two hard jets we see that the merged sample shows an additional shift towards larger azimuthal tagging jet separation. The reason is that with a third jet recoiling against the hard top or stop pair system we now have a choice to pick the two tagging jets. We systematically bias the selection towards an effectively larger $\Delta \eta_{j j}$ separation translating into more back-to-back tagging jets. However, this shift mostly affects the $\cos \Delta \phi$ distribution, while the critical $\cos (2 \Delta \phi)$ mode is symmetric around $\Delta \phi=\pi / 2$ and therefore just slightly tilted. The fact that for top pair production the kinematic effect from additional jet radiation looks similar to the $\cos \Delta \phi$ mode from spin correlations explains the surprising finding of Ref. [23] that the parton shower simulation seems to capture some of the expected spin correlations while it should not.

The size of $A_{2}$ is only slightly affected by the different simulational approaches shown in Table II, i.e. the theorydriven unmerged 2-jet setup and the more realistic merged 3 -jet case. If anything, the effect in $\cos (2 \Delta \phi)$ is more pronounced in the multijet case, contrary to what is observed as azimuthal decorrelation in 2-jet production. The two stop mass benchmarks are consistent with each other. Already for $\Delta \eta_{j j}>2$ we observe the expected sign difference between the fermionic and scalar processes. It will become an experimental issue how wide a rapidity separation of the two tagging jets is needed to extract the most information with a limited sample size.

\section{STOP SEARCHES}

The results obtained in the last section at parton level and using Monte-Carlo truth clearly demonstrate the analytic argument of Sec. II. Once all helicity information is taken into account and kinematic cuts restrict events to the VBF 
phase space, the stop events have a positive coefficient $A_{2}$, while the top background has a negative $A_{2}$. However, these results do not yet demonstrate that this difference between scalars and fermions can be used to enhance the stop sample among tops in a real experiment. One might worry that the identification of the tagging jets, combinatorics, or detector effects could wash out these correlations and make them experimentally invisible.

To confirm the experimental accessibility of the azimuthal correlation as a way to separate top pairs from stop pairs we now hadronize the parton level event samples with PYTHIA and apply the fast detector simulation DELPHES3 [33] with configuration files provided by the Snowmass Energy Frontier simulations [34]. Jets are clustered using the anti- $k_{T}$ [27] algorithm with $R=0.5$. All decays are included via PYTHIA, so we do not systematically account for spin correlations and interference patterns in the production and decay processes. From the last section it is clear that the details of the top and stop decays play no role in our analysis, beyond triggering and combinatorial challenges. In our analysis we include both semileptonic and dileptonic top pair decays. Fully hadronic decays of tops could be added once we resolve QCD and combinatorical issues, discussed for example in Ref. [35].

We generate the equivalent of $4.8 \mathrm{fb}^{-1}$ of $14 \mathrm{TeV}$ LHC data for the top background and both stop signal points. Although this is much less than the planned integrated luminosity of the next stage of LHC running, generating the corresponding full data set would be extremely resource intensive and not essential for purposes of demonstrating the feasibility of the $\Delta \phi$ technique. Indeed, as we will show below, even with only $\sim 5 \mathrm{fb}^{-1}$, the interference effect can already make stops known in the top sample, though additional luminosity would be required to improve the statistical significance.

Depending on the assumed decay channel we require one or two electrons and muons, required to have

$$
p_{T, \ell}>20 \mathrm{GeV} \text { and }\left|\eta_{\ell}\right|<2.5 \text {. }
$$

Regardless of the selection criteria of forward jets, we require exactly two $b$-tagged jets with

$$
p_{T, b}>50 \mathrm{GeV} \text { and }\left|\eta_{b}\right|<2.5,
$$

using the DELPHES 3 efficiency of approximately $70 \%$ per $b$ tag. For the upcoming $14 \mathrm{TeV}$ runs of the LHC, where pileup and jet energy calibration might be an issue, we follow two potential choices for the jet requirements:

$$
\begin{array}{ll}
\text { (1) } p_{T, j}>20 \mathrm{GeV} \text { and } & \left|\eta_{j}\right|<2.5 \text {, } \\
\text { (2) } p_{T, j}>20 \mathrm{GeV} \text { and } & \left|\eta_{j}\right|<4.5 \text {. }
\end{array}
$$

While the conservative assumption will prove to be sufficient to reveal the presence of degenerate stops, 
TABLE III. Cumulative efficiencies, including branching ratios, after detection selection criteria, in both dileptonic and semileptonic channels. Also shown is the cross section after all cuts are applied. The "Leptons" cut requires two (one) $e$ or $\mu$ for the dilepton (semileptonic) channel. Two $b$-tagged and two (four) or more non- $b$ tagged jets are required to pass " $b$-tag $\&$ jets," and the semileptonic $W$-mass reconstruction is defined in the text. The final $|\Delta \eta|$ criteria is applied for both jet selection criteria as defined in Eq. (13).

\begin{tabular}{llcccc}
\hline \hline & & \multicolumn{2}{c}{$\left|\eta_{j}\right|<2.5,\left|\Delta \eta_{j j}\right|>2$} & \multicolumn{2}{c}{$\left|\eta_{j}\right|<4.5,\left|\Delta \eta_{j j}\right|>3$} \\
& & Dileptonic & Semileptonic & Dileptonic & Semileptonic \\
\hline $\bar{t} \bar{t}$ & Leptons & $3.2 \%$ & $29 \%$ & $3.2 \%$ & $29 \%$ \\
& $+b$-tag \& jets & $0.17 \%$ & $0.98 \%$ & $0.23 \%$ & $1.5 \%$ \\
& $+W$ mass & $\ldots$ & $0.19 \%$ & $\ldots$ & $0.25 \%$ \\
& $+|\Delta \eta|$ & $0.053 \%$ & $0.066 \%$ & $0.061 \%$ & $0.064 \%$ \\
& Final $\sigma$ & $505 \mathrm{fb}$ & $629 \mathrm{fb}$ & $582 \mathrm{fb}$ & $610 \mathrm{fb} \%$ \\
$\tilde{t}^{*}(175,1)$ & Leptons & $3.3 \%$ & $29 \%$ & $3.3 \%$ & $29 \%$ \\
& $+b$-tag \& jets & $0.14 \%$ & $0.87 \%$ & $0.19 \%$ & $1.3 \%$ \\
& $+W$ mass & $\ldots$ & $0.17 \%$ & $\ldots$ & $0.23 \%$ \\
& $+|\Delta \eta|$ & $0.041 \%$ & $0.060 \%$ & $0.048 \%$ & $0.058 \%$ \\
& Final $\sigma$ & $55 \mathrm{fb}$ & $81 \mathrm{fb}$ & $65 \mathrm{fb}$ & $78 \mathrm{fb}$ \\
& Leptons & $3.3 \%$ & $29 \%$ & $3.3 \%$ & $29 \%$ \\
$\tilde{t} t^{*}(200,25)$ & $+b$-tag \& jets & $0.17 \%$ & $1.1 \%$ & $0.23 \%$ & $1.6 \%$ \\
& $+W$ mass & $\ldots$ & $0.22 \%$ & $\ldots$ & $0.28 \%$ \\
& $+|\Delta \eta|$ & $0.050 \%$ & $0.076 \%$ & $0.057 \%$ & $0.069 \%$ \\
& Final $\sigma$ & $36 \mathrm{fb}$ & $55 \mathrm{fb}$ & $41 \mathrm{fb}$ & $50 \mathrm{fb}$ \\
\hline \hline
\end{tabular}

including tagging jets to $|\eta|<4.5$ will improve the physics reach in this type of search.

For the dileptonic channel, we require two or more light-flavor jets. In the semileptonic channel we require four or more jets. Due to limited statistics, in the dileptonic channel we do not subdivide the events into different lepton flavor combinations, though this could be useful for a full experimental analysis. Similarly, a full experimental analysis might find it useful to include a systematic multijet analysis for tagging jets as well as decay jets [36], but in this paper we limit ourselves to the cleanest possible signature.

To differentiate the $W$-decay jets from the VBF tagging jets in the semileptonic channel, we suggest the following reconstruction algorithm: of all pairs of central $\left(\left|\eta_{j}\right|<1\right)$ jets passing a staggered cut $p_{T, j}>60,30 \mathrm{GeV}$ we take the pair with an invariant mass closest to $m_{W}$. If an event has such a pair of jets and their invariant mass is within $30 \mathrm{GeV}$ of the $m_{W}$, it is retained for the VBF selection criteria. The two highest- $p_{T}$ QCD jets remaining must then have an invariant mass of either less than $50 \mathrm{GeV}$ or greater than $100 \mathrm{GeV}$, to avoid possible misidentification with the $W$-boson decay products. This strict set of requirements provides a very clean sample of events where the two VBF jets are well separated from all other hadronic activity in the detector, though the efficiency is correspondingly low, and improvements on this algorithm are obviously possible.

The highest- $p_{T}$ non- $W$-tagged jets in the semileptonic sample and the highest- $p_{T}$ jets in dileptonic events are likely be the two tagging jets, so we apply the $\Delta \eta_{j j}$ cut. In the conservative jet selection scenario (1) with $\left|\eta_{j}\right|<2.5$ we only require $\left|\Delta \eta_{j j}\right|>2$, in order not to cut too deeply into the efficiency. For the more optimistic situation (2) with $\left|\eta_{j}\right|<4.5$ we can also require a larger jet separation: $\left|\Delta \eta_{j j}\right|>3$. From all events passing this final cut we construct the $\Delta \phi$ distribution. The final efficiencies and effective cross sections for both the dileptonic and semileptonic channels are shown in Table III, including the efficiencies of each cut leading up to the final $\Delta \eta$ selection.

Based on the $4.8 \mathrm{fb}^{-1}$ of simulated signal and background data given in Table IV, we can extrapolate what integrated luminosities would be required to observe a significant number of stop pair events inside the top sample. Clearly, the statistical errors from $5 \mathrm{fb}^{-1}$ of integrated luminosity would be too large to make any statement, as the difference between the background distribution and the background plus signal is equivalent to the fit uncertainties.

However, by taking the central fit values of the $d \sigma / \Delta \phi$ differential distribution as the "true" parameter values, we can determine the statistical power for a given amount of data. The luminosity from the first year of LHC14 running is expected to be around $25 \mathrm{fb}^{-1}$. This data set would reduce the statistical errors on the $A_{2} / A_{0}$ parameter to approximately $1 \%$. This would allow a $\sim 1.5 \sigma$ statistical differentiation between background and background plus signal for $175 \mathrm{GeV}$ tops in the dileptonic channel $(\sim 1 \sigma$ for $200 \mathrm{GeV}$ stops) in the current detector configuration, and somewhat less in the semileptonic channel. With improved jet tracking in the forward region, this might be improved to $1.7 \sigma$ with a year's luminosity. With a data set of $100 \mathrm{fb}^{-1}, 3.2 \sigma$ observation would be possible in both channels for $175 \mathrm{GeV}$ stops, and $2 \sigma$ discovery for $200 \mathrm{GeV}$ stops, assuming the conservative $|\eta|$ requirements. This would be improved to $3.7 \sigma$ for $175 \mathrm{GeV}(2.4 \sigma$ for $200 \mathrm{GeV})$ stops assuming the detector performance allows for $|\eta|<4.5$ in the tagging jets. 
TABLE IV. Best-fit values for the $\cos (2 \Delta \phi)$ coefficients $A_{2}$, normalized to the constant term $A_{0}$, defined in Eq. (6), for dileptonic and semileptonic events corresponding to $4.8 \mathrm{fb}^{-1}$ of luminosity, after fast detector simulation. Fits to the two stop signal points are performed for signal only as well as signal plus top background.

\begin{tabular}{lccccc}
\hline \hline & & \multicolumn{2}{c}{$\left|\eta_{j}\right|<2.5,\left|\Delta \eta_{j j}\right|>2$} & \multicolumn{2}{c}{$\left|\eta_{j}\right|<4.5,\left|\Delta \eta_{j j}\right|>3$} \\
& & Dileptonic $A_{2} / A_{0}$ & Semileptonic $A_{2} / A_{0}$ & Dileptonic $A_{2} / A_{0}$ & Semileptonic $A_{2} / A_{0}$ \\
\hline $\bar{t}$ & & $-0.10 \pm 0.03$ & $-0.05 \pm 0.03$ & $-0.12 \pm 0.03$ & $-0.08 \pm 0.03$ \\
$t \bar{t}^{*}(175,1)$ & $\bar{t}^{*}$ only & $+0.20 \pm 0.09$ & $+0.10 \pm 0.07$ & $+0.16 \pm 0.09$ & $+0.18 \pm 0.07$ \\
& $\tilde{t}^{*} \tilde{t}^{*}+t \bar{t}$ & $-0.07 \pm 0.03$ & $-0.03 \pm 0.02$ & $-0.09 \pm 0.03$ & $-0.05 \pm 0.02$ \\
$t \bar{t}^{*}(200,25)$ & $t \bar{t}^{*}$ only & $+0.22 \pm 0.11$ & $+0.03 \pm 0.08$ & $+0.18 \pm 0.11$ & $+0.16 \pm 0.10$ \\
& $\tilde{t} \tilde{t}^{*}+\bar{t} \bar{t}$ & $-0.08 \pm 0.03$ & $-0.04 \pm 0.01$ & $-0.10 \pm 0.03$ & $-0.06 \pm 0.03$ \\
\hline \hline
\end{tabular}

Such statements do not include systematic errors, which are clearly of concern for an observable so dependent on jet reconstruction and identification. However, analysis of tagging jets has already been proven to work in Higgs studies with the $8 \mathrm{TeV}$ run. Moreover, as noted in this paper, several handles are available to allow experimental control of these issues. The signal will be visible in both semileptonic and dileptonic decays, and with sufficient luminosity the dileptonic channel could be further broken down into the different flavor combinations. The turn-on of the nontrivial $A_{2}$ signal as the $\Delta \eta$ cut is instituted provides an important cross-check, and it is possible that selection cuts intended to isolate the $\beta$ dependence [7] of the top and stop signals will also define useful sidebands.

\section{CONCLUSIONS}

During the first LHC run tagging jets have been shown to be powerful tools in observing Higgs decays to photons, $W$ boson, and tau leptons. In the coming LHC runs with almost twice the collider energy their role will become even more pronounced, also reaching beyond Higgs analyses. Similar to the spin and $C P$ studies based on weak-boson-fusion Higgs events [8,9], we can test top quark properties in top pair production with two forward jets $[7,11]$. This tagging jet analysis has the general advantage that it does not rely on the reconstruction of the hard process, in our case the top pair. Instead, we can use the dependence on the azimuthal angle $\Delta \phi$ between the tagging jets to search for nonstandard events in the top sample at the LHC. Specifically, the coefficient $A_{2}$ of the $\cos (2 \Delta \phi)$ term in the distribution is negative for top pair production, whereas light scalar top pairs will give a significant positive contribution to this observable.

We first showed how the different signs can be understood in terms of the gluon helicity combinations contributing to the total rate. We then established and tested a nonstandard MADGRAPH5 setup which allows us to simulate events with all angular correlations between the ISR tagging jets intact. Using this modified generation tool we showed that the precision on the extraction of $A_{2}$ increases with the rapidity separation of the tagging jets. We also saw that the $A_{2}$ mode is not sensitive to the details of the ISR tagging jet simulation and the model parameters in the stop decays. Finally, we estimated that such an analysis should give $>3 \sigma$ results in multiple channels with around 100 inverse femtobarns of data at a $14 \mathrm{TeV}$ LHC. Because the analysis is purely based on the tagging jets it can be generalized to any hard process in and beyond the standard model.

\section{ACKNOWLEDGMENTS}

We would like to thank Stefan Prestel for checking that our MADGRAPH5 simulation makes sense. M. B. would like to thank Maria Spiropulu, Joe Lykken, Yuri Gershtein, and John-Paul Chou for helpful discussion and resources. M. B. and M. J. R. M. thank the Aspen Center for Physics, where this project was originally conceived. Finally, T. P. would like to thank Frank Krauss for deep insights into azimuthal decorrelation. This work was supported in part by U.S. Department of Energy Contract No. DESC0011095 (M. J. R. M.).
[1] D. E. Morrissey, T. Plehn, and T. M. P. Tait, Phys. Rep. 515, 1 (2012).

[2] G. Aad et al. (ATLAS Collaboration), Eur. Phys. J. C 72, 2237 (2012); J. High Energy Phys. 11 (2012) 094; Phys. Rev. Lett. 109, 211803 (2012); Phys. Lett. B 720, 13 (2013); Phys. Rev. Lett. 109, 211802 (2012); J. High
Energy Phys. 10 (2013) 189; Report No. ATLAS-CONF2013-024; Report No. ATLAS-CONF-2013-037; Report No. ATLAS-CONF-2013-048; Report No. ATLASCONF-2013-065.

[3] S. Chatrchyan et al. (CMS Collaboration), Report No. CMSPAS-SUS-12-005; Report No. CMS-PAS-SUS-12-030; 
Report No. CMS-PAS-SUS-13-004; Report No. CMS-PASSUS-13-011.

[4] D. S. M. Alves, M. R. Buckley, P. J. Fox, J. D. Lykken, and C.-T. Yu, Phys. Rev. D 87, 035016 (2013).

[5] G. Aad et al. (ATLAS Collaboration), Report No. ATLASCONF-2013-068; S. Chatrchyan et al. (CMS Collaboration), Report No. CMS-PAS-SUS-13-009.

[6] S. Bornhauser, M. Drees, S. Grab, and J. S. Kim, Phys. Rev. D 83, 035008 (2011); N. Desai and B. Mukhopadhyaya, J. High Energy Phys. 05 (2012) 057; Z. Han, A. Katz, D. Krohn, and M. Reece, J. High Energy Phys. 08 (2012) 083; C. Kilic and B. Tweedie, J. High Energy Phys. 04 (2013) 110; G. Belanger, R. M. Godbole, L. Hartgring, and I. Niessen, J. High Energy Phys. 05 (2013) 167; X.-Q. Li, Z.-G. Si, K. Wang, L. Wang, L. Zhang, and G. Zhu, Phys. Rev. D 89, 077703 (2014); E. L. Berger, Q.-H. Cao, J.-H. Yu, and H. Zhang, Phys. Rev. Lett. 109, 152004 (2012); arXiv:1305.7266.

[7] K. Hagiwara and S. Mukhopadhyay, J. High Energy Phys. 05 (2013) 019.

[8] O. J. P. Eboli and D. Zeppenfeld, Phys. Lett. B 495, 147 (2000); T. Plehn, D. L. Rainwater, and D. Zeppenfeld, Phys. Rev. Lett. 88, 051801 (2002); V. Hankele, G. Klamke, and D. Zeppenfeld, arXiv:hep-ph/0605117; V. Hankele, G. Klamke, D. Zeppenfeld, and T. Figy, Phys. Rev. D 74, 095001 (2006); C. Ruwiedel, N. Wermes, and M. Schumacher, Eur. Phys. J. C 51, 385 (2007); K. Hagiwara, Q. Li, and K. Mawatari, J. High Energy Phys. 07 (2009) 101; A. Djouadi, R. M. Godbole, B. Mellado, and K. Mohan, Phys. Lett. B 723, 307 (2013).

[9] C. Englert, D. Goncalves-Netto, K. Mawatari, and T. Plehn, J. High Energy Phys. 01 (2013) 148.

[10] V. Del Duca, W. Kilgore, C. Oleari, C. Schmidt, and D. Zeppenfeld, Nucl. Phys. B616, 367 (2001); J. R. Andersen, K. Arnold, and D. Zeppenfeld, J. High Energy Phys. 06 (2010) 091; Phys. Rev. D 84, 095025 (2011); C. Englert, D. Goncalves, G. Nail, and M. Spannowsky, Phys. Rev. D 88, 013016 (2013); F. Campanario and M. Kubocz, Phys. Rev. D 88, 054021 (2013); arXiv:1402.1154.

[11] M. R. Buckley and M. J. Ramsey-Musolf, J. High Energy Phys. 09 (2011) 094.

[12] G. Aad et al. (ATLAS Collaboration), Phys. Rev. Lett. 106, 172002 (2011).

[13] V. Khachatryan et al. (CMS Collaboration), Phys. Rev. Lett. 106, 122003 (2011).

[14] H. Baer, M. Drees, R. Godbole, J. F. Gunion, and X. Tata, Phys. Rev. D 44, 725 (1991); H. Baer, J. Sender, and X. Tata, Phys. Rev. D 50, 4517 (1994).

[15] G. Aad et al. (ATLAS Collaboration), Eur. Phys. J. C 71, 1577 (2011); J. High Energy Phys. 05 (2012) 059; Phys. Lett. B 717, 89 (2012); Eur. Phys. J. C 73, 2261 (2013); 73, 2328 (2013).

[16] S. Chatrchyan et al. (CMS Collaboration), Phys. Rev. D 85, 112007 (2012); J. High Energy Phys. 11 (2012) 067; 05 (2013) 065; Phys. Lett. B 720, 83 (2013); Eur. Phys. J. C 73, 2386 (2013).

[17] G. Aad et al. (ATLAS Collaboration), Report No. ATLASCONF-2013-097.

[18] S. Chatrchyan et al. (CMS Collaboration), Report No. CMSPAS-TOP-12-006; Report No. CMS-PAS-TOP-12-007.
[19] T. A. Aaltonen et al. (CDF and D0 Collaborations), Phys. Rev. D 89, 072001 (2014).

[20] M. Czakon, P. Fiedler, and A. Mitov, Phys. Rev. Lett. 110, 252004 (2013).

[21] W. Beenakker, M. Krämer, T. Plehn, M. Spira, and P. M. Zerwas, Nucl. Phys. B515, 3 (1998); for further improvements see e.g. W. Hollik, M. Kollar, and M. K. Trenkel, J. High Energy Phys. 02 (2008) 018; A. Kulesza and L. Motyka, Phys. Rev. D 80, 095004 (2009); W. Beenakker, S. Brensing, M. Kramer, A. Kulesza, E. Laenen, and I. Niessen, J. High Energy Phys. 08 (2010) 098; M. Beneke, P. Falgari, and C. Schwinn, Nucl. Phys. B842, 414 (2011).

[22] R. Kleiss and W. J. Stirling, Phys. Lett. B 200, 193 (1988); U. Baur and E. W. N. Glover, Phys. Lett. B 252, 683 (1990); V. D. Barger, K. Cheung, T. Han, J. Ohnemus, and D. Zeppenfeld, Phys. Rev. D 44, 1426 (1991).

[23] T. Plehn, D. Rainwater, and P. Z. Skands, Phys. Lett. B 645, 217 (2007).

[24] J. Alwall, M. Herquet, F. Maltoni, O. Mattelaer, and T. Stelzer, J. High Energy Phys. 06 (2011) 128.

[25] M. L. Mangano, M. Moretti, and R. Pittau, Nucl. Phys. B632, 343 (2002).

[26] T. Sjostrand, S. Mrenna, and P. Z. Skands, J. High Energy Phys. 05 (2006) 026.

[27] M. Cacciari, G. P. Salam, and G. Soyez, J. High Energy Phys. 04 (2008) 063; Eur. Phys. J. C 72, 1896 (2012).

[28] J. Alwall, https://cp3.irmp.ucl.ac.be/projects/madgraph/ wiki/IntroMatching.

[29] R. Boughezal and M. Schulze, Phys. Rev. Lett. 110, 192002 (2013); Phys. Rev. D 88, 114002 (2013).

[30] J. Alwall, S. de Visscher, and F. Maltoni, J. High Energy Phys. 02 (2009) 017; J. Alwall, K. Hiramatsu, M. M. Nojiri, and Y. Shimizu, Phys. Rev. Lett. 103, 151802 (2009); M. M. Nojiri and K. Sakurai, Phys. Rev. D 82, 115026 (2010).

[31] G.-C. Cho, K. Hagiwara, J. Kanzaki, T. Plehn, D. Rainwater, and T. Stelzer, Phys. Rev. D 73, 054002 (2006); J. Alwall, D. Rainwater, and T. Plehn, Phys. Rev. D 76, 055006 (2007); G. F. Giudice, T. Han, K. Wang, and L.-T. Wang, Phys. Rev. D 81, 115011 (2010); A. G. Delannoy, B. Dutta, A. Gurrola, W. Johns, T. Kamon, E. Luiggi, A. Melo, P. Sheldon et al., Phys. Rev. Lett. 111, 061801 (2013); U. Haisch, A. Hibbs, and E. Re, Phys. Rev. D 89, 034009 (2014).

[32] T. Plehn and T. M. P. Tait, J. Phys. G 36, 075001 (2009).

[33] J. de Favereau, C. Delaere, P. Demin, A. Giammanco, V. Lemaître, A. Mertens, and M. Selvaggi (DELPHES 3 Collaboration), J. High Energy Phys. 02 (2014) 057.

[34] J. Anderson et al., arXiv:1309.1057; A. Avetisyan et al., arXiv:1308.0843; arXiv:1308.1636.

[35] T. Plehn, M. Spannowsky, M. Takeuchi, and D. Zerwas, J. High Energy Phys. 10 (2010) 078; M. R. Buckley, T. Plehn, and M. Takeuchi, J. High Energy Phys. 08 (2013) 086; M. R. Buckley, T. Plehn, T. Schell, and M. Takeuchi, J. High Energy Phys. 02 (2014) 130.

[36] C. Bernaciak, M. S. A. Buschmann, A. Butter, and T. Plehn, Phys. Rev. D 87, 073014 (2013); C. Bernaciak, B. Mellado, T. Plehn, X. Ruan, and P. Schichtel, Phys. Rev. D 89, 053006 (2014). 